\title{
Prevalence and Pattern of COVID-19 among Healthcare Workers in Rivers State Nigeria
}

\author{
Datonye Dennis Alasia $^{* *}$ (D), Omosivie Maduka2 ${ }^{(1)}$ \\ ${ }^{1}$ Departments of Internal Medicine, University of Port Harcourt, Port Harcourt, Nigeria \\ ${ }^{2}$ Preventive and Social Medicine, University of Port Harcourt, Port Harcourt, Nigeria \\ Email: *datonye.alasia@uniport.edu.ng
}

How to cite this paper: Alasia, D.D. and Maduka, O. (2021) Prevalence and Pattern of COVID-19 among Healthcare Workers in Rivers State Nigeria. Occupational Diseases and Environmental Medicine, 9, 20-32. https://doi.org/10.4236/odem.2021.91003

Received: January 3, 2021

Accepted: February 19, 2021

Published: February 22, 2021

Copyright $\odot 2021$ by author(s) and Scientific Research Publishing Inc. This work is licensed under the Creative Commons Attribution International License (CC BY 4.0).

http://creativecommons.org/licenses/by/4.0/

\begin{abstract}
Introduction: The evaluation of COVID-19 prevalence among healthcare workers (HCW) within the general population of COVID-19 cases is an important epidemiologic variable. The objective of this study is to describe the prevalence and patterns of COVID-19 infection in HCWs amongst a group of patients receiving care for COVID-19 in Rivers state, Nigeria. Methods: This study was a prospective descriptive study of all consenting patients who received care through hospitals, designated for COVID-19 treatment in Rivers state either as in-patient or out-patient following a laboratory-confirmed diagnosis of COVID-19 based on a positive SARS-CoV-2 RT-PCR from April to September 2020. Results: A total number of 646 COVID-19 patients were enrolled over the study period with 98 (15.2\%) HCWs in the patient population. The HCWs with COVID-19 consisted largely of Doctors 47 (47.9\%), Nurses 30 (30.6\%), and socio-sanitary and hygiene workers 10 (10.2\%). There were 46 (46.9\%) female HCWs, compared to Non-HCWs with 112 (21.1\%), females, $p=0.000$. Sixty-eight (69.4\%) HCWs had a source of contact for infection established compared to Non-HCWs with an established source of contact in 181 (34.2\%), $\mathrm{p}=0.000$. Eight $(8.2 \%)$ HCWs had Severe disease compared to $52(9.8 \%)$ Non-HCWs with severe disease, $\mathrm{p}=0.670$. The case fatality in HCWs was $1 \%$ compared to $1.9 \%$ in Non-HCWs, $\mathrm{p}=0.554$. Conclusion: The prevalence of COVID-19 among HCWs in the study location is high with clinical and clinical support staff particularly, doctors and nurses are at higher risk of COVID-19 infection. This calls for action to improve and prevent HCWs infections in hospital settings in addition to improving HCW infection prevention behaviour in the community. The intensification of risk communication, provision of protective equipment (PPE), and training on the appropriate use of PPE; in addition to routine surveillance for infection is recommended.
\end{abstract}




\section{Keywords}

SARS-CoV-2 Infection, COVID-19, Prevalence, Healthcare Workers, Nigeria

\section{Introduction}

The response to the COVID-19 pandemic as declared by the World Health Organisation (WHO) in March 2020 [1], elevated global cognizance of the role of healthcare workers as a critical resource for the world. This acknowledgement was accentuated as healthcare workers (HCWs) became frontline combatants across all pillars of the COVID-19 response with the attendant risk of infection. Healthcare worker infection, therefore, became an issue of concern in the early period of the pandemic response with documentation of alarming rates of HCW infections [2] [3]. A report from the WHO joint mission to China in February 2020 reported 2055 COVID-19 laboratory-confirmed cases of HCW healthcare infections in 476 hospitals across China [2]. Correspondingly Wang et al. [3], reported that $29 \%$ of patients with COVID-19 infection were HCWs from a cohort of 138 patients treated in a hospital in Wuhan. The study [3] also referred to the risk of widespread transmission in healthcare settings as evidenced by a super spreader patient who infected over $10 \mathrm{HCW}$ in the hospital. Similar observations regarding HCW infections were also noted in Spain as of 31st March 2020 with over $9400 \mathrm{HCW}$ consisting of approximately 15\% of all confirmed cases infected with COVID-19 [4]. The WHO Africa region office also reported that over 10,000 HCWs had been infected with COVID-19 in Africa as of July 2020 , with an average rate of $10 \%$ of infections in some key countries [5].

The evaluation of healthcare worker's prevalence among the general population of COVID-19 cases has therefore become an important variable in the epidemiologic analysis of the pandemic; with studies around the world documenting a range of $3 \%-19 \%$ prevalence of HCWs among the populations infected with SARS-CoV-2 [6] [7] [8] [9]. Wu et al. [10] in a Chinese centre for disease control (CDC) report, documented that $3.8 \%$ of 44,672 cases were healthcare workers; while two studies [11] [12] from Italy reported that HCWs accounted for 9\% [11] and 9.8\% [12] of cases in March 2020. Elimian et al. [13] in descriptive epidemiology of COVID-19 in Nigeria, found that HCWs accounted for 9.3\% of all positive cases. A hospital prevalence study from Qatar [6] reported a prevalence of $10.6 \%$ among tested HCWs; whereas the USA CDC [7] reported a $19 \%$ prevalence of COVID-19 in HCWs among a population of 49,370 people. In addition to the established higher risk of reporting a positive test for COVID-19 among frontline HCWs compared to the general population [14]; patterns of distinctions in disease demographics and epidemiology, clinical trends and outcomes have also been documented in comparisons of HCWs and the general population with COVID-19 [3] [7] [8] [10]. The evaluation of HCW infections and applicable epidemiologic patterns at subnational and national le- 
vels is, therefore, an important research focus; as the consequence of HCWs infection is a depletion in the workforce available to confront the pandemic and increase risk of transmission among other HCWs and patients attending hospitals. These shortages in the health workforce result from self-isolation of health workers for periods of at least two weeks and the time lost to ill health thus imposing an increased workload on available staff. Besides, health workplace safety may also be compromised by the risk of hospital-acquired infections from healthcare workers to patients. The objective of this study is to describe the prevalence and patterns of COVID-19 infection in HCWs amongst a group of patients receiving care for COVID-19 through ambulatory and in-patient hospital services in Rivers state, Nigeria.

\section{Methodology}

\subsection{Study Location}

The study was conducted in Rivers State, one of Nigeria's 36 states located in south-south, Nigeria. The state ranks within the top 7 in the number of COVID-19 cases in the country as stated by the Nigerian Centre for disease control (NCDC) since June 2020 [15].

\subsection{Study Design, and Population}

This study was a prospective descriptive study of all consenting patients who received care through hospitals, designated for COVID-19 treatment in Rivers state either as in-patient or ambulatory (out-patient) following a laboratory-confirmed diagnosis of COVID-19 based on a positive SARS-CoV-2 RT-PCR after presentation with suggestive symptoms or contact tracing of other patients from April to September 2020. The patients were categorised based on their occupation into Healthcare workers and Non-Healthcare workers. The healthcare workers were classified based on the WHO and International Labour Organisation (ILO) International Standard Classification of occupations (ISCO) [16], and their roles in patient management and healthcare services into six groups. Health Professional (HP) Group 1-Medical and dental doctors; Health Professional (HP) Group 2-Nurses; Health and Health Associate Professional (H \& HAP) Group 3-(Pharmacist; Laboratory scientist and technologist, clinical psychologist, social support services and medical records information); Health and Health Associate Professional (H \& HAP) Group 4-(Water Sanitation \& Hygiene (WASH)/Socio-sanitary/Hygienist, Health attendants, Respiratory and anaesthetic technicians); Health and Health Associate Professional (H \& HAP) Group 5-(Public Health officers, epidemiology and disease surveillance officers) and Health Management and Health Support Personnel (HM \& HSP) Group 6-(Administrative and support staff and hospital managers).

\subsection{Data Collection}

A data extraction form built on the open data kit (ODK) tool was used to collect 
data that was subsequently exported to a Microsoft Excel spreadsheet. Data domains included socio-demography, epidemiology, symptomatology, comorbidity, and disease outcome. Disease severity was classified using Nigerian Centre for disease control National COVID-19 case management guideline parameters [17] as severe and non-severe, with severity defined presence of fever $>38^{\circ} \mathrm{C}$ or suspected respiratory infection, plus one of respiratory rate $>30$ breaths $/ \mathrm{min}$; severe respiratory distress; $>\mathrm{SpO}_{2} \leq 93 \%$ on room air \& Presence of co-morbid conditions such as diabetes, asthma, hypertension in adults and cough or difficulty in breathing \& at least one of the following central cyanosis or $\mathrm{SpO}_{2}<92 \%$; severe respiratory distress e.g. grunting breathing, very severe chest in-drawing \& signs of pneumonia in children. Disease outcome was classified into discharged and died.

\subsection{Ethical Considerations}

The Ethical approval was obtained from the Research Ethics Committee of the University of Port Harcourt Teaching Hospital, Rivers state before the commencement of the study. Confidentiality was maintained by the removal of patient identifiers from the dataset and ensuring that only researchers involved in this study had access to the extracted data.

\subsection{Statistical Analysis}

The data was exported from the Microsoft Excel spreadsheet into IBM Statistical Package for Social Sciences (SPSS) version 23 for the data analysis. The proportion of HCWs among the cohort and the distribution of HCWs by professional category was done using basic descriptive statistics and frequencies. The HCWs were then compared with all adult patients aged over 18 years in the cohort for patterns in epidemiologic and clinical variables using both descriptive and inferential analysis. An independent t-test was used for comparison of means for categorical variables. Qualitative variables were compared for proportions in the occurrence of socio-demographic, epidemiological, and clinical characteristics between HCWs and non HCWs using Pearson chi-square test with mantel Hensel correction with relative risk and odds ratio as appropriate. A two-tailed p-value less than 0.05 was considered statistically significant. The population size for the study was time-bound and dependent on the course of the pandemic. This accounts for limitations arising from the sample size.

\section{Results}

A total number of 646 patients were enrolled over the study period with 98 (15.2\%) HCWs in the patient population.

The HCWs consisted largely of HP Group 1-Doctors 47 (47.9\%), HP Group 2-Nurses 30 (30.6\%), H \& HAP Group 3-5 (5.1\%), H \& HAP Group 4-10 (10.2\%), H \& HAP Group 5-3 (3.1\%), HM \& SP Group 6-3 (3.03\%). The distribution of HCWs is displayed in Table 1 and Figure 1. 


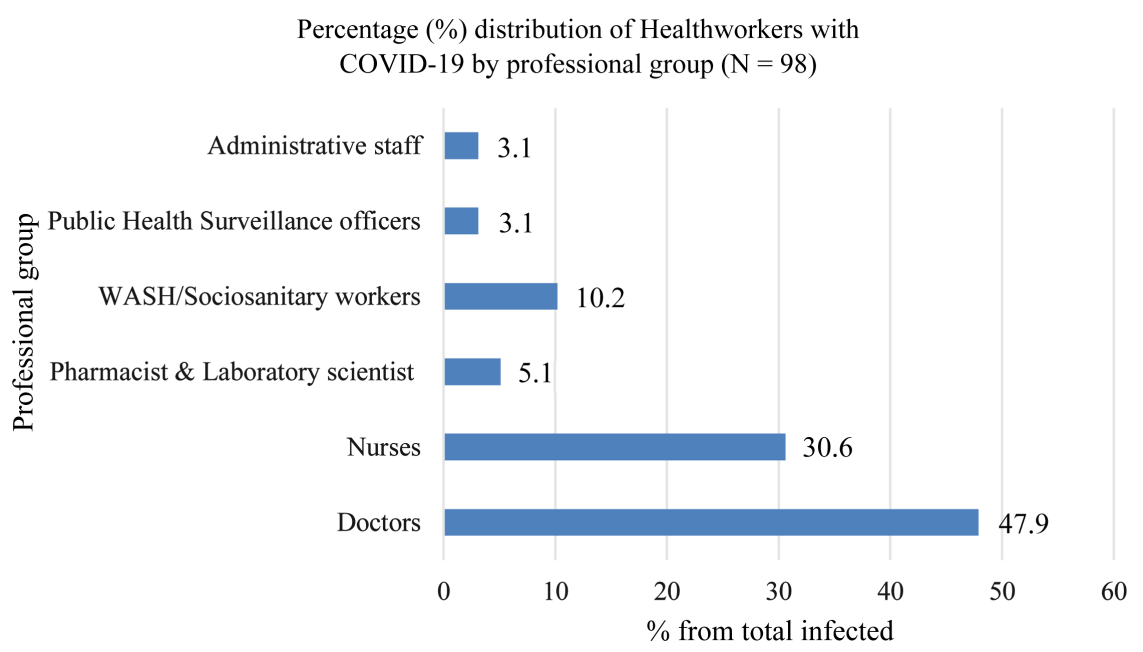

Figure 1. Distribution of health workers with COVID-19 by professional groups.

Table 1. Distribution of healthcare workers with COVID-19 by professional grouping.

\begin{tabular}{|c|c|c|c|c|}
\hline Health Professional Group & Specific Profession & $\mathrm{N}$ & $\begin{array}{c}\text { Group } \\
\text { total }\end{array}$ & $\begin{array}{c}\text { Percentage } \\
\%\end{array}$ \\
\hline Health Professional Group 1 & Doctors & 47 & 47 & 47.9 \\
\hline Health Professional Group 2 & Nurses & 30 & 30 & 30.6 \\
\hline Health \& Health Associate & Pharmacist & 1 & & \\
\hline Professional Group 3 & Laboratory Science/Technologist & 4 & 5 & 5.1 \\
\hline $\begin{array}{l}\text { Health \& Health Associate } \\
\text { Professional Group } 4\end{array}$ & $\begin{array}{c}\text { WASH/Sociosanitary \& } \\
\text { Environmental health officer }\end{array}$ & 10 & 10 & 10.2 \\
\hline $\begin{array}{l}\text { Health \& Health Associate } \\
\text { Professional Group } 5\end{array}$ & Public Health Surveillance officers & 3 & 3 & 3.1 \\
\hline \multirow[t]{2}{*}{$\begin{array}{c}\text { Health Management \& } \\
\text { Support Professional Group } 6\end{array}$} & Administrative staff & 3 & 3 & 3.1 \\
\hline & Total & & 98 & $100 \%$ \\
\hline
\end{tabular}

Age: The mean age of $98 \mathrm{HCWs}$ was $40.22 \pm 11.17$ compared to 530 patients $\geq$ 18 years with $39.89 \pm 11.95, \mathrm{p}$-value $=0.798$ (see Table 2$)$.

The age group distribution of HCWs, with comparison to Non-HCWs, is as displayed in Table 2. The majority of the HCWs were in the $31-40(40.8 \%)$ and $41-50(23.5 \%)$ year age groups, there was no significant difference in comparison with non-healthcare workers $\mathrm{p}=0.202, \chi^{2}=9.777$ (see Table 2).

Gender: There were 52 (53.1\%) male HCWs and 46 (46.9\%) female HCWs, compared to Non HCWs with 418 (78.9\%) males and 112 (21.1\%), female proportion, $\mathrm{p}=0.000, \chi^{2}=29.903$.

The pattern of contact source, comorbidity, disease severity, and outcome variables are presented in Table 3. Contact source: 68 (69.4\%) HCWs had a source of contact for infection established while a source of contact was unknown for 30 (30.6\%) HCWs; compared to Non-HCWs with an established source of contact in $181(34.2 \%)$ and $349(65.8 \%)$ with the source of contact unknown, $p=0.000$, 
Table 2. Age group and gender distribution of healthcare workers compared with non-healthcare workers.

\begin{tabular}{|c|c|c|c|c|c|c|}
\hline \multirow{2}{*}{$\begin{array}{c}\text { Variable } \\
\text { Age Group }\end{array}$} & \multicolumn{2}{|c|}{ Healthcare Workers } & \multicolumn{2}{|c|}{ Non-Healthcare Workers } & \multirow{2}{*}{$x^{2}$} & \multirow{2}{*}{$\mathrm{p}$ value } \\
\hline & $\mathbf{N}$ & $\%$ & $\mathbf{N}$ & $\%$ & & \\
\hline $18-30$ & 17 & 17.3 & 124 & 23.4 & & \\
\hline $31-40$ & 40 & 40.8 & 173 & 32.6 & 9.777 & 0.202 \\
\hline $41-50$ & 23 & 23.5 & 131 & 24.7 & & \\
\hline $51-60$ & 9 & 9.2 & 77 & 14.5 & & \\
\hline $61-70$ & 8 & 8.2 & 19 & 3.6 & & \\
\hline$>70$ & 1 & 1.0 & 6 & 1.1 & & \\
\hline Total & 98 & 100 & 530 & 99.9 & & \\
\hline Mean Age (Years) & \multicolumn{2}{|c|}{$40.22 \pm 11.168$} & \multicolumn{2}{|c|}{$39.89 \pm 11.949$} & & 0.798 \\
\hline \multirow[t]{2}{*}{ Gender } & $\begin{array}{c}\text { Male } \\
(\%)\end{array}$ & $\begin{array}{c}\text { Female } \\
\text { N (\%) }\end{array}$ & $\begin{array}{c}\text { Male } \\
\text { N (\%) }\end{array}$ & $\begin{array}{c}\text { Female } \\
\mathrm{N}(\%)\end{array}$ & & \\
\hline & $53.1 \%$ & $46.9 \%$ & $78.9 \%$ & $21.1 \%$ & 29.903 & 0.000 \\
\hline
\end{tabular}

Table 3. The pattern of comorbidity, disease severity, and outcome variables.

\begin{tabular}{ccccccc}
\hline \multirow{2}{*}{ Variable } & \multicolumn{7}{c}{ Healthcare workers } & \multicolumn{2}{c}{ Non-Healthcare workers } & \multirow{2}{*}{$\mathbf{N}=\mathbf{5 3 0}$} & \multirow{2}{*}{ p value } \\
\cline { 2 - 5 } & Yes (\%) & No (\%) & Yes (\%) & No (\%) & & \\
\hline Contact known & 69.4 & 30.6 & 34.2 & 65.8 & 43.881 & 0.000 \\
Comorbidity & 33.7 & 66.3 & 33.6 & 66.4 & 0.554 & 0.758 \\
Hypertension & 20.4 & 79.2 & 25.7 & 74.3 & 1.531 & 0.465 \\
Diabetes & 10.2 & 89.8 & 7.74 & 92.3 & 1.072 & 0.585 \\
Asthma & 2.0 & 98.0 & 0.9 & 99.1 & 1.282 & 0.525 \\
Heart disease & 2.0 & 98.0 & 0.8 & 99.2 & 0.444 & 0.801 \\
Kidney Disease & 1.0 & 97.0 & 0.6 & 99.4 & 0.643 & 0.725 \\
HIV/AID & 0.0 & 100.0 & 0.4 & 99.6 & 0.737 & 0.692 \\
COPD & 0.0 & 100.0 & 0.4 & 99.6 & 2.554 & 0.466 \\
Severe disease & 8.2 & 91.8 & 9.8 & 91.2 & 0.670 & 0.802 \\
Death & 1.0 & 99.0 & 1.9 & 98.1 & 1.179 & 0.554 \\
\hline
\end{tabular}

$\chi^{2}=43.881$, odds ratio $=4.43, \mathrm{CI}=2.378-7.061$. Sixty-one $(62.2 \%)$ of the HCWs had their source of contact within the hospital while all the Non-HCWs had a source of contact for infection in the community.

Presence of comorbidity: Thirty three (33.7\%) HWCs had at least one comorbidity present while 65 (66.3\%) had none; compared to Non-HCWs with 178 (33.5\%) who had a comorbidity and $352(66.4 \%)$ with none, $\mathrm{p}=0.758, \chi^{2}=$ 0.554 .

Disease severity: 8 (8.2) HCWs had severe disease and 90 (91.8\%) had non-severe disease compared to Non-HCWs with $52(9.8 \%)$ with severe disease and 478 (91.2\%) with non-severe disease, $\mathrm{p}=0.670, \chi^{2}=0.802$.

Case fatality: One (1.0\%), HCW died while 97 (99.0\%) were discharged com- 
pared to $10(1.9 \%)$ who died and $520(98.1 \%)$, who were discharged, $\mathrm{p}=0.554, \chi^{2}$ $=1.179$.

Comorbidities: The pattern of comorbidities is as displayed in Table 3. The leading comorbidities in HCWs were hypertension $20.4 \%$, diabetes $10.2 \%$, and asthma $2.0 \%$. Hypertension $25.7 \%$ and diabetes $7.7 \%$ were also the leading comorbidities in non-healthcare workers. There were no significant differences in the proportions of comorbidity in both groups. The pattern of symptoms: The pattern of symptoms is shown in Table 4 and Figure 2. The leading symptoms

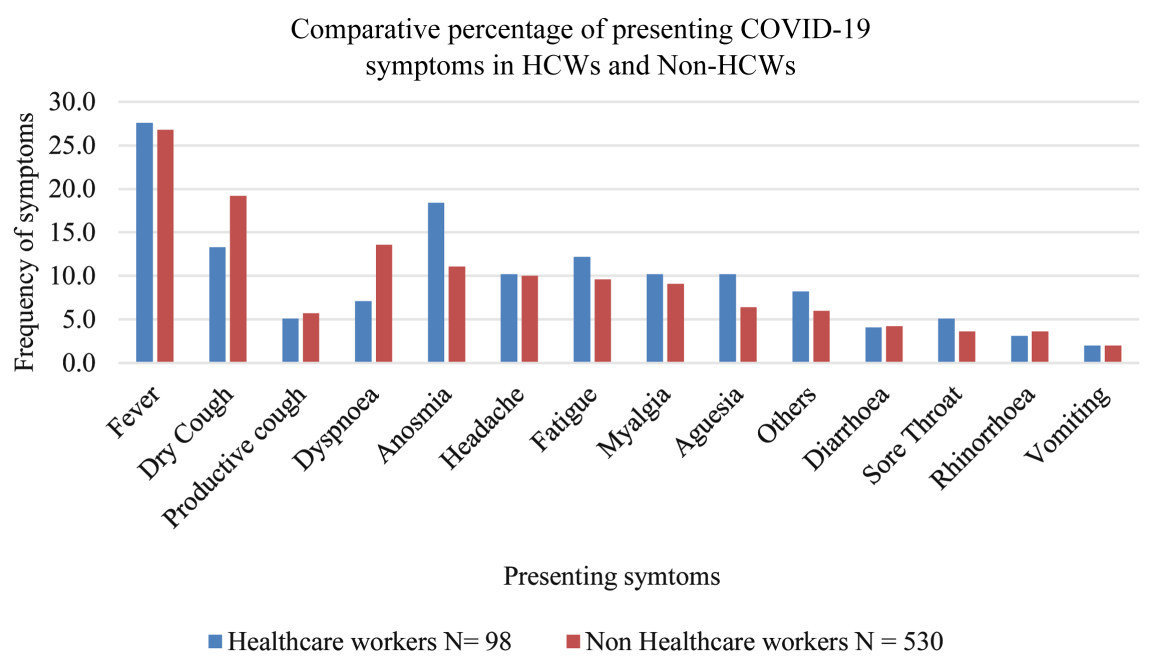

Figure 2. The pattern of presenting symptoms in patients with COVID-19.

Table 4. The pattern of symptoms among HCWs and Non-HCWs with COVID-19.

\begin{tabular}{|c|c|c|c|c|c|c|}
\hline & \multicolumn{2}{|c|}{$\begin{array}{l}\text { Healthcare } \\
\qquad \mathrm{N}=98\end{array}$} & \multicolumn{2}{|c|}{$\begin{array}{l}\text { Non Healthcare } \\
\qquad \mathrm{N}=530\end{array}$} & \multirow[t]{2}{*}{$x^{2}$} & \multirow[t]{2}{*}{$\mathrm{p}$ value } \\
\hline & $\mathbf{N}$ & $\%$ & $\mathbf{N}$ & $\%$ & & \\
\hline \multicolumn{7}{|l|}{ Symptom } \\
\hline Fever & 27 & 27.6 & 142 & 26.8 & 0.403 & 0.818 \\
\hline Dry Cough & 13 & 13.3 & 102 & 19.2 & 2.287 & 0.319 \\
\hline Productive cough & 5 & 5.1 & 30 & 5.7 & 0.411 & 0.814 \\
\hline Dyspnoea & 7 & 7.1 & 72 & 13.6 & 3.646 & 0.302 \\
\hline Anosmia & 18 & 18.4 & 59 & 11.1 & 4.974 & 0.174 \\
\hline Headache & 10 & 10.2 & 53 & 10.0 & 0.374 & 0.830 \\
\hline Fatigue & 12 & 12.2 & 51 & 9.62 & 1.029 & 0.598 \\
\hline Myalgia & 10 & 10.2 & 48 & 9.1 & 0.510 & 0.775 \\
\hline Aguesia & 10 & 10.2 & 34 & 6.4 & 0.2239 & 0.326 \\
\hline Others & 8 & 8.2 & 32 & 6.0 & 1.197 & 0.550 \\
\hline Diarrhoea & 4 & 4.1 & 22 & 4.2 & 0.553 & 0.759 \\
\hline Sore Throat & 5 & 5.1 & 19 & 3.6 & 1.083 & 0.582 \\
\hline Rhinorrhoea & 3 & 3.1 & 19 & 3.6 & 0.616 & 0.863 \\
\hline Vomiting & 2 & 2.0 & 10 & 2.0 & 0.564 & 0.754 \\
\hline
\end{tabular}


in HCWs were fever (27.6\%), anosmia (18.4\%), dry cough (13.3\%), fatigue (12.2\%), headaches (10.2\%), and myalgia (10.2\%), and ageusia (10.2\%). In Non-Healthcare workers, the leading symptoms were fever (26.79\%), dry cough (19.2\%), shortness of breath (13.6\%), anosmia (11.1\%), headache (10.0\%), fatigue $(9.6 \%)$, and myalgia $(9.1 \%)$. There were no significant differences in the proportion of symptom patterns.

\section{Discussion}

Healthcare worker infection with SARS-CoV-2 has been a global source of concern since the onset of the pandemic with alarming prevalence rates of $\mathrm{HCW}$ infection. The prevalence of HCWs infection in this group of patients seen in hospitals from a Nigerian state was $15.2 \%$. Though is it within the range of $3 \%$ $19 \%$ reported globally [6] [7] [8] [9]; it is a source of concern as it is higher than the $9.3 \%$ prevalence reported by Elimian et al. [13], in a descriptive study of COVID-19 from all states in Nigeria. The understanding that Rivers state is one of the high burden states for COVID-19 infection may also explain the higher prevalence of HCWs infection above a National average value in this study. The prevalence of HCW infection in this study is similar to $15 \%$ reported in Spain [4] and lower than 19\% from the USA [7], which were both within the early phases of the pandemic in March and April 2020 respectively. In further comparison with other studies, the prevalence of HCWs with COVID-19 in this study is above $2.8 \%$ and $2.5 \%$ observed by Shararidad et al. [18] and Giesen et al. [19] from Iran and Spain respectively among hospitalised patients. A systematic review of global studies [8] reported HCW infection prevalence of 3.9\% consisting of an estimated 152,888 of 3,912,156 cases as of 8 may 2020; while Wu et al. [10] from China found a prevalence of 3.4\%. Two other studies [11] [12], from Italy reported a prevalence of $9 \%$ and $9.8 \%$ respectively while a study in Qatar [6] found a prevalence of $10 \%$. The prevalence of the above studies, is lower than the finding in this index study. It is therefore evident that HCWs contribute significantly to the burden of COVID-19 in the study location with prevalence rates above what is general observed from many other studies.

In the professional group of HCWs the most affected by COVID-19 in this study were doctors (47.9\%), nurses (30.6\%), and WASH/Environmental health, health attendants $(10.2 \%)$. This shows that medical and clinical staff who have direct contact with patients and support staff who are in contact with the patient's environment are most at risk for infection. This pattern corresponds with the findings of Zheng et al. [20] in a study from the London teaching hospital which found that clinical staff groups had higher infection rates $7.3 \%$ compared to non-clinical staff with $2.8 \%$, with medical and dental and nursing and midwifery as the professional groups with the highest rates of infections. A similar pattern was also observed by Sotgui et al. [21] at an Italian forefront hospital in a serologic prevalence study for SARS-CoV-2 with doctors (47.0\%), Nurses $(26.2 \%)$, and socio-sanitary workers $(5.5 \%)$, having the highest prevalence of 
SARS-CoV-2 infection. Other studies have also corroborated this pattern as shown in a systematic review of global studies [8] which had nurses (38.6\%) and Doctors $(31.3 \%)$ as the leading professional category in correspondence with the findings of this study. Lombardi et al. [22] in Italy also reported Doctors, Health technicians, Nurses, and Health assistants $10.5 \%, 9.4 \%, 8.4 \%$, and $8 \%$ were the leading professional groups with SARS-CoV-2 infection. Fusco et al. [11] also reported a higher proportion of nurses (50\%) and doctors (23\%) in their cohort. Alajmi et al. [6] from a Qatar national surveillance study reported the highest infection rates in Nurses (33.2\%) and non-clinical support staff with $(31.3 \%)$ with physicians consisting 5\% of infections. Maskari et al. [23] from Oman reported Nurses with $38 \%$ of infections while doctors and paramedics had $13 \%$ of infections each with administrative/support staff making up 36\%. The pattern of reported by Alajmi [6] and Maskari [23] differs slightly from our pattern with nurses, non-clinical support, and paramedics having higher rates of infections compared to doctors. The variations may be due to a higher proportion of community-acquired infections over hospital-acquired infections documented in those studies. The summary of all studies still shows that clinical workers especially doctors and nurses and support staff with contact to patient environments have a higher risk of infection. Clinical staff are therefore at higher risk and require an emphasis on risk communication prevention messages, provision of PPEs, and surveillance for infection. There were no significant differences in the mean age and age group distribution profile of HCWs and non HCWs in this study, with the mean age of 40.22 years above the national average of or mean of 37.1 years explained by the exclusion of people under 18 in the comparisons. Similar age means and median and age group distribution have also been reported by other studies [8] [11] [21] [23].

There was a significant difference in the gender distribution between healthcare and non-healthcare workers in this study, with a higher female prevalence among healthcare workers compared to non-healthcare workers, this reflects the high preponderance of females in healthcare occupations in Nigeria especially nursing which accounted for over $30 \%$ of the HCWs and doctors. Other studies show a similar trend of female HCWs proportions above general population figures with Fusco et al. [11], Bandyopadhyay [8], Lombardi et al. [22], Maskari et al. [23] reporting proportions of $49 \%, 71.6 \%, 62.4 \%$, and $64 \%$ respectively.

In this study, the majority of HCWs (69.4\%) with COVID-19 had a source of contact established compared to non-healthcare workers with a predominantly unknown source of disease indicating higher levels of community transmission in Non-HCWs. Also, the majority of HCWs had their contacts within the hospital environment from patients and other healthcare workers. These findings correspond with that of Wang et al. [3] who reported a higher rate of hospital-associated transmission in HCW of $29 \%$ compared to $12.3 \%$ in hospitalized non-HCWs. This finding shows the need for better infection prevention and control practice and appropriate PPE use among HCWs in this environment to reduce transmission of SARS-CoV-2 among HCWs. There was no significant 
difference in the presence of comorbidity, the proportion of disease severity, and case fatality in the study among HCWs and non HCWs. Hypertension and diabetes were the leading comorbidities both in HCWs and Non-HCWs. This observation is reassuring as HCWs do not have a higher risk of adverse outcomes compared to the general population. Also, the case fatality among HCWs reported in this study corresponds with global observations from a systematic review by Bandyopadhyay et al. [8] that reported a global case fatality among HCWs at 1 in $100(1 \%)$. The pattern of hypertension and diabetes as the leading comorbid disease condition has also been reported by other studies [3] [23] [24]. Wang et al. [3] reported hypertension and diabetes as the leading comorbid disease conditions, while Maskari et al. [23] reported diabetes as the leading comorbid disease condition over hypertension. The range of comorbidity presence of $22.9 \%$ to $46.4 \%$ among HCWs reported by Wang et al. [3] and Maskari et al. [23] respectively is comparable to the $33.7 \%$ reported in this study.

The pattern of symptoms among the HCWs in this study was similar and did not differ significantly from non-HCWS, with fever, dry cough, fatigue, headaches, myalgia, anosmia, ageusia, and shortness of breath as the leading symptoms in line with the existing symptom pattern and other studies involving the general population [25] and HCWs [3] [6]. The presence of anosmia among HCWs as the second most common symptom in this study is a finding of interest as anosmia is predictive of less severe disease, reduced hospitalizations', and lower in-hospital mortality in COVID-19 patients [26] [27].

\section{Conclusion}

The study has shown that the prevalence of COVID-19 among HCWs in the study location is high and a cause of epidemiologic concern as HCWs contribute a significant burden of COVID-19 infections. This calls for action to improve and prevent HCWs infections in hospital settings in addition to improving HCW infection prevention behaviour in the community. Clinical and clinical support staff particularly doctors and nurses are at higher risk of COVID-19 infections and require intensification of risk communication, provision of protective equipment (PPE), and training on the appropriate use of PPE; in addition to routine surveillance for infection. There is no risk for the development of more severe disease and higher case fatality among HCWs compared to the general population.

\section{Acknowledgements}

We acknowledge all members of the health team who cared for the patients and the data collectors engaged in the study.

\section{Conflicts of Interest}

The authors declare no conflicts of interest regarding the publication of this paper. 


\section{References}

[1] WHO. WHO Director-General's Opening Remarks at the Media Briefing on COVID-19-11 March 2020.

https://www.who.int/director-general/speeches/detail/who-director-general-s-openi ng-remarks-at-the-media-briefing-on-covid-19---11-march-2020

[2] WHO-China Joint Mission. Report of the WHO-China Joint Mission on Coronavirus Disease 2019 (COVID-19), February 2020.

https://www.who.int/docs/default-source/coronaviruse/who-china-joint-mission-o n-covid-19-final-report.pdf

[3] Wang, D., Hu, B., Hu, C., Zhu, F., Liu, X., Zhang, J., et al. (2020) Clinical Characteristics of 138 Hospitalized Patients with 2019 Novel Coronavirus-Infected Pneumonia in Wuhan, China. Journal of the American Medical Association, 323, 1061. https://doi.org/10.1001/jama.2020.1585

[4] Benavides, L. (2019) The Coronavirus Crisis: Spain's Health Staff Are Catching the Coronavirus as Protective Gear Runs Short.

https://www.npr.org/sections/coronavirus-live-updates/2020/03/31/824654965/spai ns-health-staff-are-catching-the-coronavirus-as-protective-gear-runs-short

[5] WHO Regional Office for Africa (2020) Over 10000 Health Workers in Africa Infected with COVID-19. WHO Africa News 23rd July 2020.

https://www.afro.who.int/news/over-10-000-health-workers-africa-infected-covid-1 $\underline{9}$

[6] Alajmi, J., Jeremijenkoa, A.M., Abrahama, J.C., Alishaqa, M., Concepciona, E.G., Butta, A.A. and Abou-Samra, A.-B. (2020) COVID-19 Infection among Healthcare Workers in a National Healthcare System: The Qatar Experience. International Journal of Infectious Diseases, 100, 386-389.

https://doi.org/10.1016/j.ijid.2020.09.027

[7] CDC (2020) COVID-19 Response Team. Characteristics of Health Care Personnel with COVID-19: United States, February 12-April 9, 2020. Morbidity and Mortality Weekly Report, 69, 477-481. https://doi.org/10.15585/mmwr.mm6915e6

[8] Bandyopadhyay, S., Baticulon, R.E., Kadhum, M., Alser, M., Ojuka, D.K., Badereddin, Y., et al. (2020) Infection and Mortality of Healthcare Workers Worldwide from COVID-19: A Systematic Review. BMJ Global Health, 5, e003097. https://doi.org/10.1101/2020.06.04.20119594

[9] Ali, S., Noreen, S., Farooq, I., Bugshan, A. and Vohra, F. (2020) Risk Assessment of Healthcare Workers at the Frontline against COVID-19. Pakistan Journal of Medical Sciences, 36, S99-S103. https://doi.org/10.12669/pjms.36.COVID19-S4.2790

[10] Wu, Z. and MacGoogan, J.M. (2020) Characteristics of and Important Lessons from the Coronavirus Disease 2019 (COVID-19) Outbreak in China Summary of a Report of 72314 Cases from the Chinese Center for Disease Control and Prevention. JAMA, 323, 1239-1242. https://doi.org/10.1001/jama.2020.2648

[11] Fusco, F.M., Pisaturo, M., Iodice, V., Bellopede, R., Tambaro, O. and Parrella, G. (2020) COVID-19 among Healthcare Workers in a Specialist Infectious Disease Setting in Naples, Southern Italy: Results of a Cross-Sectional Surveillance Study. Journal of Hospital Infection, 105, 596-600. https://doi.org/10.1016/j.jhin.2020.06.021

[12] Livingston, E. and Bucher, K. (2020) Coronavirus Disease 2019 (COVID-19) in Italy. JAMA, 323, 1335. https://doi.org/10.1001/jama.2020.4344

[13] Elimian, K.O., Ochu, C.L., Ilori, E., Oladejo, J., Igumbor, E., Steinhardt, L., Wagai, J., et al. (2020) Descriptive Epidemiology of Coronavirus Disease 2019 in Nigeria, 27 
February-6 June 2020. Epidemiology and Infection, 148, e208. https://doi.org/10.1017/S095026882000206X

[14] Nguyen, L.H., Drew, D.A., Graham, M.S., Joshi, A.D., Guo, G.-C. and Ma, W. (2020) Risk of COVID-19 among Front-Line Health-Care Workers and the General Community: A Prospective Cohort Study. Lancet Public Health, 5, e475-e483.

[15] Nigerian Centre for Disease Control (NCDC) (2020) COVID-19 in Nigeria: Confirmed COVID-19 Cases by State, 20th November 2020. https://covid19.ncdc.gov.ng

[16] WHO (2020) Classifying Health Workers: Mapping Occupations to the International Standard Classification: International Labour Organization, International Standard Classification of Occupations: ISCO-08.

https://www.who.int/hrh/statistics/Health_workers_classification.pdf

[17] Nigerian Centre for Disease Control (NCDC) (2020) National Interim Guidelines for Clinical Management of COVID19.

https://covid19.ncdc.gov.ng/media/files/National_Interim_Guidelines_for_Clinical _Management_of_COVID-19_v3.pdf

[18] Shahriarirad, R., Khodamoradi, Z., Erfani, A., Hosseinpour, H., Ranjbar, K., Emami, Y., Mirahmadizadeh, A., et al. (2020) Epidemiological and Clinical Features of 2019 Novel Coronavirus Diseases (COVID-19) in the South of Iran. BMC Infectious Diseases, 20, 427. https://doi.org/10.1186/s12879-020-05128-x

[19] Giesen, C., Diez-Izquierdoa, L., Saa-Requejoa, C.M., Lopez-Carrilloa, I., Lopez-Vilelaa, C.A., Seco-Martineza, A. and Prieto, M.T.R. (2020) Epidemiological Characteristics of the COVID-19 Outbreak in a Secondary Hospital in Spain. American Journal of Infection Control, 49, 143-150.

[20] Zheng, C., Hafezi-Bakhtiari, N., Cooper, V., Davidson, H., Habibi, M., Riley, P. and Breathnach, A. (2020) Characteristics and Transmission Dynamics of COVID-19 in Healthcare Workers at a London Teaching Hospital. Journal of Hospital Infection, 106, 325-329. https://doi.org/10.1016/j.jhin.2020.07.025

[21] Sotgiu, G., Barassi, A., Miozzo, M., Saderi, L., Piana, A., Orfeo, N., et al. (2020) SARS-CoV-2 Specific Serological Pattern in Healthcare Workers of an Italian COVID-19 Forefront Hospital. BMC Pulmonary Medicine, 20, 203.

https://doi.org/10.1186/s12890-020-01237-0

[22] Lombardi, A., Consonni, D., Carugno, M., Bozzi, G., Mangioni, D., Muscatello, A., et al. (2020) Characteristics of 1573 Healthcare Workers Who Underwent Nasopharyngeal Swab Testing for SARS-CoV-2 in Milan, Lombardy, Italy. Clinical Microbiology and Infection, 26, 1413.e9-1413.e13.

https://doi.org/10.1016/j.cmi.2020.06.013

[23] Maskari, Z.L., Blushi, A.A.L., Khamis, F., Tai, A.A.L., Salmi, I.A.L., Harthi, H.A.L., et al. (2020) Characteristic of Healthcare Workers Infected with COVID-19, a Cross-Sectional Observational Study. International Journal of Infectious Diseases, 102, 32-36. https://doi.org/10.1016/j.ijid.2020.10.009

[24] Zhou, F., Yu, T., Du, R., Fan, G., Liu, Y., Liu, Z., et al. (2020) Clinical Course and Risk Factors for Mortality of Adult Inpatients with COVID-19 in Wuhan, China: A Retrospective Cohort Study. The Lancet, 395, 1054-1062. https://doi.org/10.1016/S0140-6736(20)30566-3

[25] Ahmed, A., Ali, A. and Hasan, S. (2020) Comparison of Epidemiological Variations in COVID-19 Patients inside and outside of China-A Meta-Analysis. Frontiers in Public Health, 8, 193. https://doi.org/10.3389/fpubh.2020.00193

[26] Talavera, B., García-Azorína, D., Martínez-Píasa, E., Trigoa, J., Hernández-Péreza, I. and Valle-Peñacoba, G. (2020) Anosmia Is Associated with Lower In-Hospital 
Mortality in COVID-19. Journal of the Neurological Sciences, 419, Article ID: 117163. https://doi.org/10.1016/j.jns.2020.117163

[27] Foster, K.J., Jauregui, E., Tajudeen, B., Faraz Bishehsari, F. and Mahdavinia, M. (2020) Smell Loss Is a Prognostic Factor for Lower Severity of Coronavirus Disease 2019. Annals of Allergy, Asthma \& Immunology, 125, 475-494.

https://doi.org/10.1016/j.anai.2020.07.023 